\title{
El arte de prevenir incendios
}

\author{
Madrigal Olmo, J. ${ }^{* 1,2}$ \\ ${ }^{\prime}$ INIA, Centro de Investigación Forestal, Dpto. Selvicultura y Gestión de los Sistemas Forestales, Laboratorio de \\ incendios forestales. Ctra. Coruña km 7,5. 28040 Madrid \\ ${ }^{2}$ iuFOR. Instituto Universitario de Gestión Forestal Sostenible uVA-INIA \\ *Autor para correspondencia: incendio@inia.es
}

\section{Resumen}

Los incendios forestales son un elemento natural en los ecosistemas Mediterráneos y el fuego ha sido utilizado como herramienta selvícola en Europa desde la Edad de Hielo. En el contexto actual de cambio global (cambio climático, cambios de usos del suelo) no parece haber una tendencia a la disminución del número de focos de ignición a pesar del aumento de la despoblación rural, con lo que los incendios podrían acelerar o desacelerar procesos en nuestros ecosistemas, con implicaciones sociales y económicas difíciles de predecir. Por tanto prevenir el impacto negativo de los incendios e integrar el fuego en la gestión se ha convertido en un verdadero reto de futuro difícil de abordar, no sólo desde el punto de vista político y de restricciones presupuestarias, sino porque existen verdaderas dudas técnicas acerca de la eficacia real de las actuaciones basadas en ciencia e ingeniería forestal. En la presente ponencia se muestran posibles opciones de futuro para mejorar la gestión de nuestros montes y prevenir incendios forestales basadas en las investigaciones de diferentes grupos nacionales e internacionales. Se discute sobre la viabilidad y eficacia de algunos tratamientos selvícolas en la prevención de grandes incendios y se proponen estrategias para la introducción del uso del fuego en los ecosistemas mediterráneos.

Palabras clave: defensa integrada contra incendios forestales, divulgación, filosofía, gestión forestal sostenible, humanidades. 


\section{1. ¿Quiénes somos de dónde venimos y a dónde vamos?}

Siempre se ha considerado que lo que diferencia a la especie humana del resto de los animales es su capacidad cognitiva. Pero cada vez hay más evidencias de que algunos animales tienen capacidad de solucionar problemas y se podrían acercar a lo que los humanos consideramos "tener consciencia de sí mismos" (Gallup 1970). Por tanto, de entre las pocas cosas objetivas que podemos considerar que diferencia a la especie humana del resto de los animales es su capacidad creativa y, en concreto, las manifestaciones artísticas y la facultad de querer explicar cómo funciona nuestro entorno, la capacidad de hacer arte y ciencia. Ambas se mantienen habitualmente en departamentos estancos en el ámbito educativo, laboral y recreativo, pero no siempre fue así. En la actualidad incluso es habitual diferenciar entre Ciencia, Arte y Humanidades, como si fueran cosas diferentes, como si la Humanidad no tuviera nada que ver con la Ciencia y el Arte o como si la Ciencia y el Arte no estuvieran pensadas y ejecutadas por seres humanos.

Y en principio fue el fuego. Efectivamente, desde una perspectiva antropológica o incluso, algunos dirían que "pyrocéntrica", el principio de la Humanidad fue la capacidad de hacer fuego. Algunos primates tienen la capacidad de diseñar herramientas pero ninguno de los que han sobrevivido a las diferentes extinciones ha tenido la capacidad de hacer fuego. Fue un descubrimiento que permitió a las comunidades nómadas calentarse y sobre todo cocinar alimentos, uno de los factores determinantes en la evolución del cerebro humano (Wrangham 2010). Además posibilitó la aparición de las primeras manifestaciones artísticas: las pinturas rupestres. Es un claro ejemplo en el que la ciencia y el arte se llevan de la mano, y en este caso el fuego es el verdadero protagonista.

La forma en la que la Humanidad se ha relacionado con el entorno natural ha cambiado, pasando de un uso y aprovechamiento de mera subsistencia a la observación de dicho entorno por el simple hecho del conocimiento o la curiosidad científica. En lugares intermedios entre una cosa y la otra andamos los ingenieros forestales intentando emplear con arte los conocimientos científicos en la mejora de los usos y aprovechamientos, para hacerlos más sostenibles en el espacio y en el tiempo, para hacerlos más compatibles con los procesos ecológicos naturales, para en definitiva, ayudar a que los sistemas forestales puedan convivir con la presencia humana y explicar cómo la presencia humana ha generado los paisajes naturales heredados hoy día.

Recientes resultados paleobotánicos muestran que el uso del fuego en Europa para la apertura de pastos para la caza se remonta a la Edad de Hielo (Kaplan et al. 2016), lo que se une a las evidencias de esta práctica ancestral en otros continentes como América o África. De nuevo el fuego como promotor de cambio en la Humanidad, en este caso de la selvicultura: el primer intento de manejo del entorno natural por parte del ser humano para su beneficio propio, el arte de quemar para subsistir.

Desvincular las decisiones técnicas de la ciencia y la creatividad nos puede llevar a decisiones correctas simplemente por la eficacia de la costumbre y las certezas 
experimentales de nuestros ancestros: "lo que siempre ha funcionado seguirá funcionando". Este precepto tiene el gran inconveniente de que son necesarias varias generaciones para adaptarse a los cambios y que nunca sabremos los "por qués", simplemente funcionaremos como artesanos de la Naturaleza y no como artistas. El artista, como el científico, no tiene la facultad de saber todas las respuestas, sino de plantear bien algunas preguntas. Pueden existir artesanos perfectos, aquellos que ejecuten a la perfección un cuerpo de doctrina, unas instrucciones heredas de padres a hijos, una forma de hacer las cosas ¿Existe el artista perfecto? ¿Existe la ciencia perfecta? ¿Existe la ciencia forestal perfecta?

Por otro lado el arte, tal como lo entendemos hoy día, como expresión intelectual de creatividad con el objeto de transmitir o provocar sensaciones en el propio artista o en el resto de la Humanidad, también se ha valido de la ciencia para avanzar en sus formas de expresión y en su vehículo de transmisión al resto de la Humanidad ¿Por qué la imprenta supuso una revolución en la literatura? ¿Hubiera avanzado la Humanidad al mismo ritmo sin este invento? ¿Por qué? ¿Ha cambiado en esencia la expresión artística literaria por el hecho de que la imprenta facilitara la difusión de la literatura a más cantidad de personas? El cine y la fotografía son disciplinas artísticas que no existirían sin la ciencia ¿qué ha supuesto de cambio para la Humanidad?

LiDAR, drones, Aeronoves no tripuladas, Láser, Rádar, satélites, sistemas de información geográfica, Big data, TICs, Modelos de simulación, son nuevas herramientas tecnológicas disponibles para la gestión forestal y la investigación pero ¿va a cambiar en esencia algo la ciencia forestal? ¿Puede la herramienta tecnológica cambiar los preceptos fundamentales de una disciplina científica? ¿Internet y la revolu-

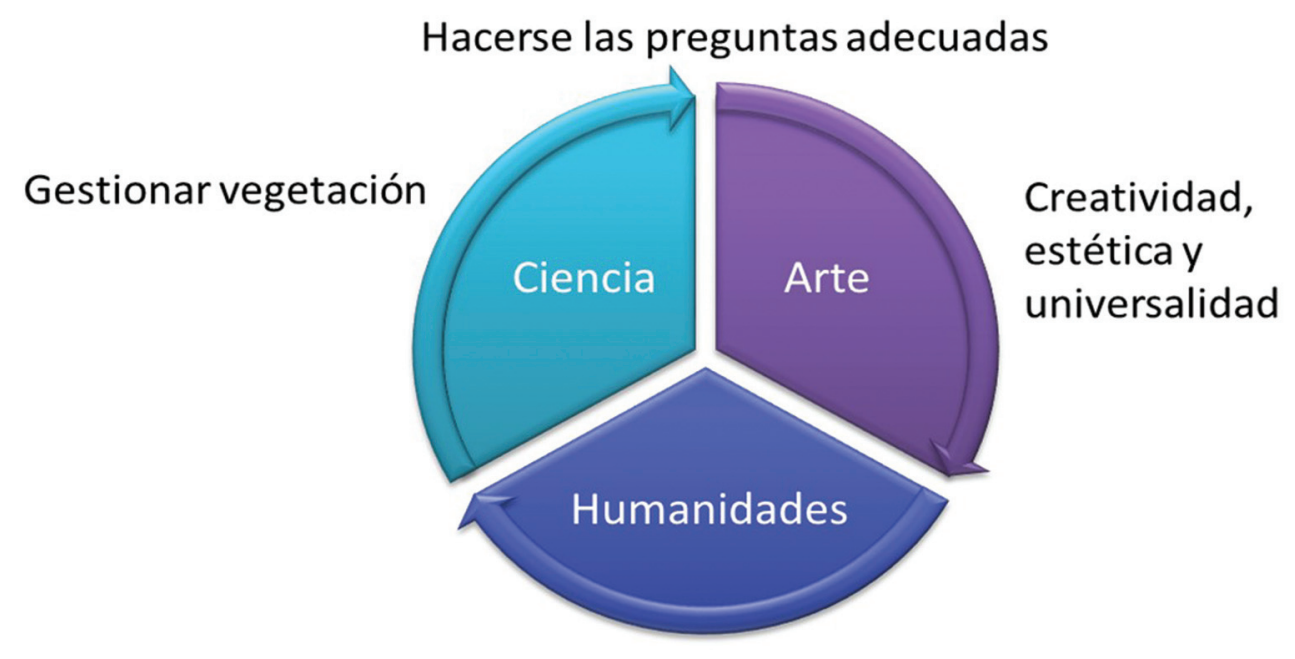

Tratar con personas

Figura 1. Modelo conceptual de prevención de incendios incluyendo ciencia, humanidades y arte entendido éste último como capacidad de realizar acciones originales de forma creativa y universal para aumentar el impacto en la sociedad y por tanto la eficacia de las medidas adoptadas. 
ción tecnológica están generando cambios en la disciplina forestal? ¿Cambia nuestra forma de ver y abordar los problemas?

Prevenir incendios en nuestros ecosistemas implica básicamente dos cosas: disminuir las fuentes de ignición de causa humana y reducir la continuidad y cantidad de combustible (Fig. 1). La primera implica el arte de tratar con las personas, por tanto incluye disciplinas de estudio claramente orientadas a lo que tradicionalmente se considera "Humanidades". Entre otras podríamos incluir la psicología, la sociología, la pedagogía, la historia, la geografía humana, la filosofía, la política, todas ellas aplicadas fundamentalmente al mundo rural o a aquella parte del mundo urbano que se acerca al monte y necesita herramientas para comprenderlo. La gestión de los combustibles tiene una dimensión más técnica y se englobaría en lo que denominamos normalmente como "Ciencias" en este caso "Ciencias forestales", en las que, como sabemos, también incluye multitud de disciplinas: ecología, selvicultura, aprovechamientos, planificación e inventario, sanidad forestal, economía, conservación e incluso ordenamiento urbanístico y territorial en el nuevo concepto de interfaz urbano-forestal y urbano-rural. Entonces ¿Cómo aunamos este conjunto de disciplinas en la resolución de un problema? ¿necesitamos actuar con arte para prevenir incendios?

\section{Interpretar la realidad}

El primer paso para actuar es saber con qué estamos trabajando. Ingeniería es el arte de actuar con ingenio para resolver un problema práctico y para ello necesitamos conocer el problema. El inventario y la biometría. Es lo que un profesor durante mi carrera nos resumía muy claramente: el arte de medir, contar y pesar. Con un objetivo muy claro, interpretar la realidad, acercarnos a ella en la medida que pueda ser explicada con un modelo o simplemente descrita a través de los datos de campo. De esto saben mucho los artistas. Nos llevan ventaja de largo porque llevan muchos siglos dedicados a ello. Nos dan su visión de la realidad. Filtrada por sus ojos y con objetivos menos prácticos y más sentimentales, pero probablemente por ello, con mayor eficacia en su cometido.

La presencia de nuevas tecnologías como el LiDAR y la disponibilidad de gran cantidad de datos online (BigData) está posibilitando interpretar la realidad con otras herramientas. Pero ¿la revolución que supuso la fotografía generó la muerte de la pintura? Estamos siendo cada vez más realistas, en el sentido pictórico de la palabra, a la hora de representar nuestros bosques $¿ E s t a m o s$ por tanto en la Era del realismo pictórico o de la fotografía? ¿Es el realismo la mejor interpretación de la realidad?

Lo cierto es que la verdadera revolución creativa en la pintura fue el expresionismo y su más ilustre antecesor, Francisco de Goya. Este autor ha sido considerado por un algoritmo matemático como el más influyente de la Historia de la pintura (Elgammal y Saleh 2015) porque es el primero que apuesta por deformar la realidad conscientemente, aun teniendo la técnica para representarla fielmente como sus antecesores realistas. Asume que no es necesario representar a la perfección para hallar la esencia de las cosas. La realidad difuminada (Fisher 2000) podría ser la mejor forma 
de interpretar porque el exceso de información nos impide llegar al meollo. La mente humana tiende a clasificar en cajones estancos para ordenar nuestras ideas. Esta sistemática nos permite proponer formas de clasificar la realidad un tanto rígidas pero útiles. Así podemos hacer mapas de vegetación, mapas de combustibles, proponer desbroces, cortafuegos en "teselas" o "trozos" del territorio fácilmente interpretables por un mapa. Pero ¿es siempre necesario clasificar todo el espacio? ¿Debemos forzar clasificaciones arbitrarias si la realidad no es así? ¿Podemos modelizar una realidad difusa, difuminada, borrosa, tal y como proponían los expresionistas? Una vez más la ciencia y el arte se unen para resolver un problema por obra de las matemáticas. La lógica difusa, la inteligencia artificial, la minería de datos, las redes neuronales, son herramientas matemáticas que permiten simular la toma de decisiones del cerebro humano. Su aplicación a las ciencias forestales (Fig. 2) es aún insuficiente, por ejemplo en la modelización de los combustibles, en la planificación de la interfaz urbano-forestal (Castillo 2012) o en el diseño de infraestructuras preventivas (Fernández-Migueláñez et al. 2014).

Cuando el profesor Keatin en "El club de los poetas muertos" (Weir 1989) invita a sus alumnos a arrancar las páginas del libro de literatura que aboga por un método capaz de medir la belleza de un poema, está también cuestionando un asunto que atañe directamente a la ciencia forestal: ¿Podemos medir la Naturaleza? Quizás esto no es posible y seguramente no es necesario, pero sí podemos ofrecer métricas razonables que nos ayuden en la toma de decisiones. Y esas métricas probablemente deberían huir del realismo o del hiperrealismo (como el que ofrece la fotografía) para obtener algo creativo. Por tanto no podemos medir la belleza, en gran parte subjetiva, pero sí podemos medir en qué medida una propuesta es novedosa respecto a lo existente con anterioridad y cómo ha sido capaz de influir en generaciones posteriores (Elgammal y Saleh 2015).

La revolución tecnológica del siglo XXI ha puesto a nuestra disposición las mejores herramientas de la Historia para interpretar la realidad pero su buen uso y su influencia futura dependerá de su flexibilidad y de la capacidad de adaptarse a los cambios, tanto de la realidad de nuestros montes como de técnicas aún no disponibles. Por tanto el arte de interpretar la realidad, como siempre, lo pone el profesional, lo pone el artista, lo pone la persona. El talento es universal e intemporal.

\section{Volver a la esencia}

Pablo Picasso fue un genio convirtiéndose en un artista muy influyente en la Historia del Arte porque tuvo la valentía y el acierto de replantearlo todo, de volver a la esencia de las cosas, de intentar mirar la realidad adulta con la mirada y la simplicidad de nuestros ancestros, de volver a la cueva de las pinturas rupestres desde la intelectualidad de miles de años de Humanidad. Otros artistas eligieron otro camino hacia lo esencial. Klein, por ejemplo, demostró que hay margen para la creación incluso en algo esencial para la pintura, como es el color, y creó el color "azul Klein" hoy día presente en multitud de objetos de diseño y moda. 

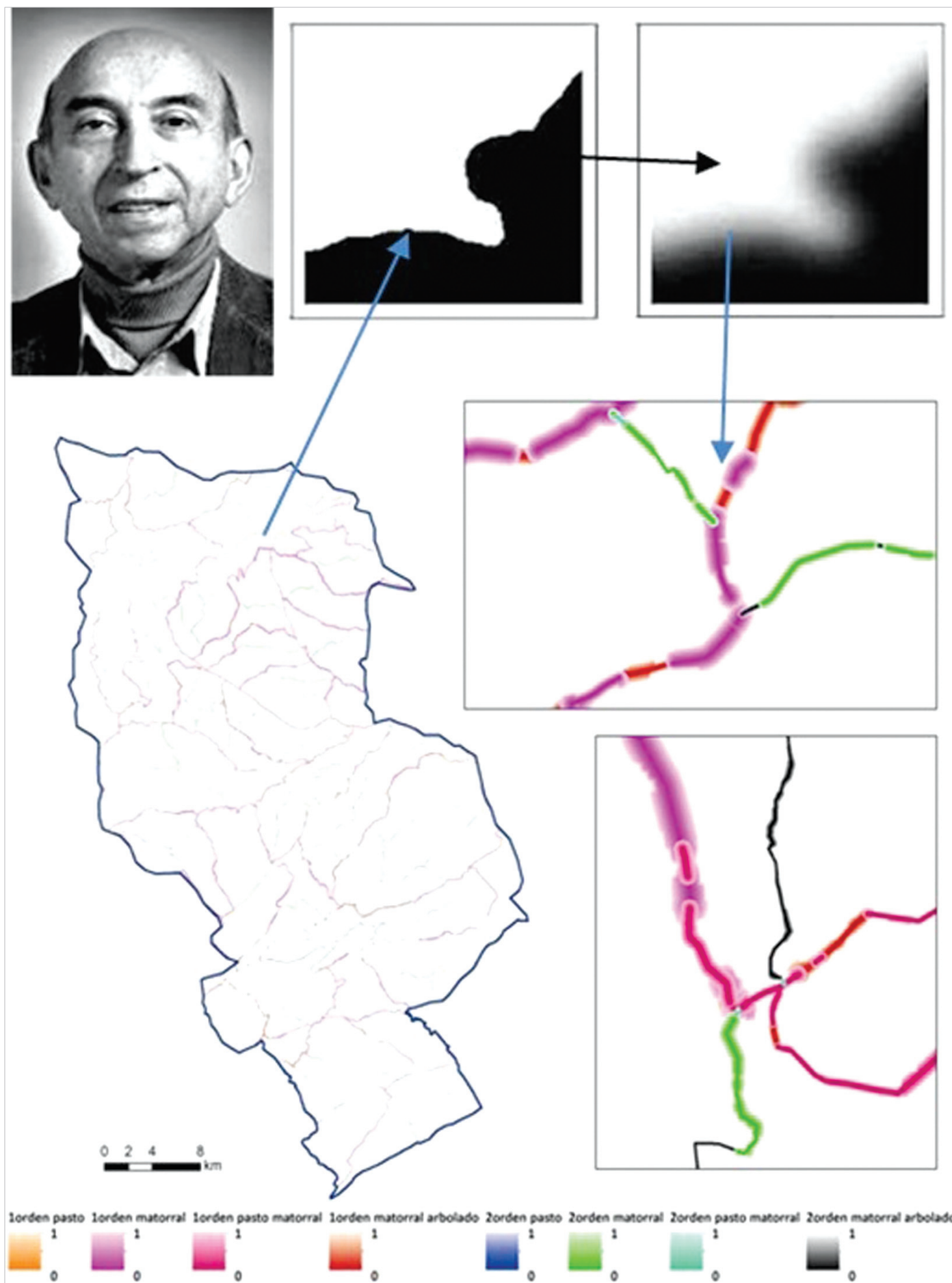

Figura 2. El matemático Lofti A. Zadeh en 1965 formuló el concepto de lógica difusa que hoy puede ser usado en geografía y cartografía (Fisher 2000) para, por ejemplo, la optimización de redes de áreas cortafuegos (Fernández-Migueláñez et al. 2014). 
El modelo de Rothermel (1972) ha supuesto una influencia vital en las estrategias de prevención y extinción de incendios, influyendo a nivel mundial en la gestión forestal en la medida que es la base de la mayoría de los simuladores de incendios usados en planificación durante la extinción pero también en la evaluación del peligro de incendios, ubicación y dimensionamiento de infraestrucuras preventivas y evaluación de la eficacia de tratamientos selvícolas y quemas prescritas. La simplicidad y elegancia de su formulación ha impregnado la formación de varias generaciones de ingenieros/as forestales especialistas en incendios. Este modelo ha dado buenos resultados durante muchos años y a pesar de las críticas recibidas, sigue siendo el modelo más usado internacionalmente. Sin embargo el cambio global obliga a nuevos retos y planteamientos. Asumir que el comportamiento del fuego va a ser estacionario, condición sine qua non para aplicar el modelo de Rothermel, se antoja cada vez menos asumible. Hay que volver a la esencia. Y probablemente ya lo hemos conseguido. La nueva teoría de pulsos de Mark Finney y colaboradores (Finney et al. 2015) tiene esa elegancia prometedora de Picasso y Klein, porque explica lo aparentemente obvio con las herramientas que ofrece la ciencia. La invención del fuego lo cambió todo y la característica principal del fuego es la presencia de la llama (Fig. 3). M. Finney ha vuelto al origen y ha dado en la diana. El tiempo dirá si este planteamiento pasa a la Historia y ofrece modelos de comportamiento ante los retos del futuro cercano en la Era del Antropoceno y el cambio global.

\section{La caverna}

La gestión de los combustibles es replantear la dicotomía entre tradición, cultura rural e innovación científica. Platón (427-347 a. C.) utilizó el mito de la caverna (Platón 2003) para diferenciar los actos humanos llevados por la tradición y la costumbre cultural respecto a aquellos procesados por el conocimiento. Plantea la cultura popular como una cárcel, una cueva oscura y misteriosa donde el individuo sólo ve la sombra de las ideas y no las ideas en sí mismas. Sólo aquel capaz de darse cuenta del engaño mediante el estudio y el conocimiento puede escapar a este hecho. Esta metáfora ha influenciado mucho el mundo de la ciencia y la filosofía puesto que ha establecido una frontera casi infranqueable entre ambos mundos que ha llevado a la ausencia de comunicación entre el pueblo llano y los sabios que controlan el conocimiento ¿nos hemos salvado hoy día de esta dicotomía?

José Saramago (1922-2010) da una vuelta de tuerca al mito en su novela "La caverna" (Saramago 2000). Muestra una situación tan decadente como real en la que un artesano es literalmente absorbido por la caverna de nuestro tiempo que son los centros comerciales, ejemplo de los productos manufacturados. Esta metáfora es claramente aplicable a nuestro mundo rural. Lo artesanal ha muerto. La actividad agraria que no sea mecanizable está condenada. Lo extensivo está denostado ¿Quedan alternativas? ¿Está condenado el mundo rural a ser un simple escaparate etnográfico para los urbanitas?

Una cuestión que está suscitando mucha controversia en los últimos tiempos es 


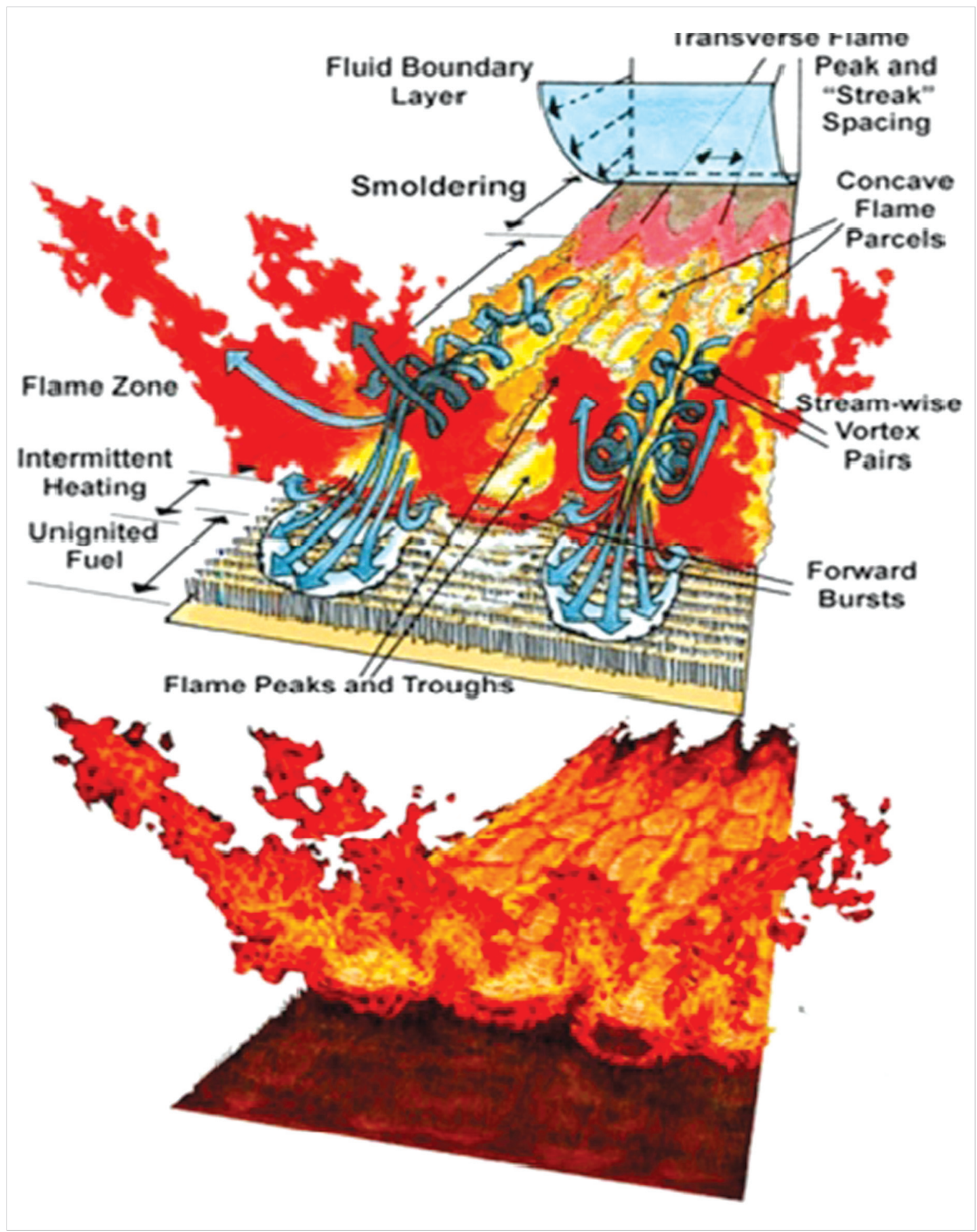

Figura 3. Infografía de la nueva teoría de pulsos para explicar el comportamientos del fuego. (Finney et al. 2015)

si realmente la selvicultura en general y la gestión del combustible en particular, sirve para prevenir incendios en el nuevo contexto de cambio global (Boer et al. 2015). La comunidad científica y técnica está siendo cada vez más pesimista en la efi- 
cacia indiscriminada de desbroces o claras, simplemente por el hecho de que hay que "ocupar" a los medios de extinción durante el invierno reduciendo carga de combustible. Estas actuaciones desgraciadamente suelen ser insuficientes y en muchos casos contrarias al criterio técnico de planificación en el espacio y en el tiempo. $\mathrm{La}$ mayoría de las propuestas coinciden en que, ante la imposibilidad de gestionar todo el territorio, deberíamos de centrarnos en nodos de propagación o puntos estratégicos de gestión donde las inversiones podrían ser más eficaces (Regos et al. 2013, 2016). Porque para actuar de verdad a nivel de paisaje, la sociedad y nuestros políticos tienen que entender que hay que volver a "lo extensivo" si queremos prevenir los grandes incendios (Marino et al. 2014; Madrigal et al. 2017). Pero recuperar cultivos agrícolas abandonados, ganadería extensiva y el uso del fuego en el siglo XXI implica la necesidad de tecnificación y de nuevos pastores, agricultores y selvicultores jóvenes que vuelvan a nuestras áreas rurales, porque la sociedad del conocimiento quiere salir de la caverna sin renunciar a los usos y aprovechamientos tradicionales, pero también manteniendo los avances tecnológicos de nuestra vida cotidiana (Fig. 4).

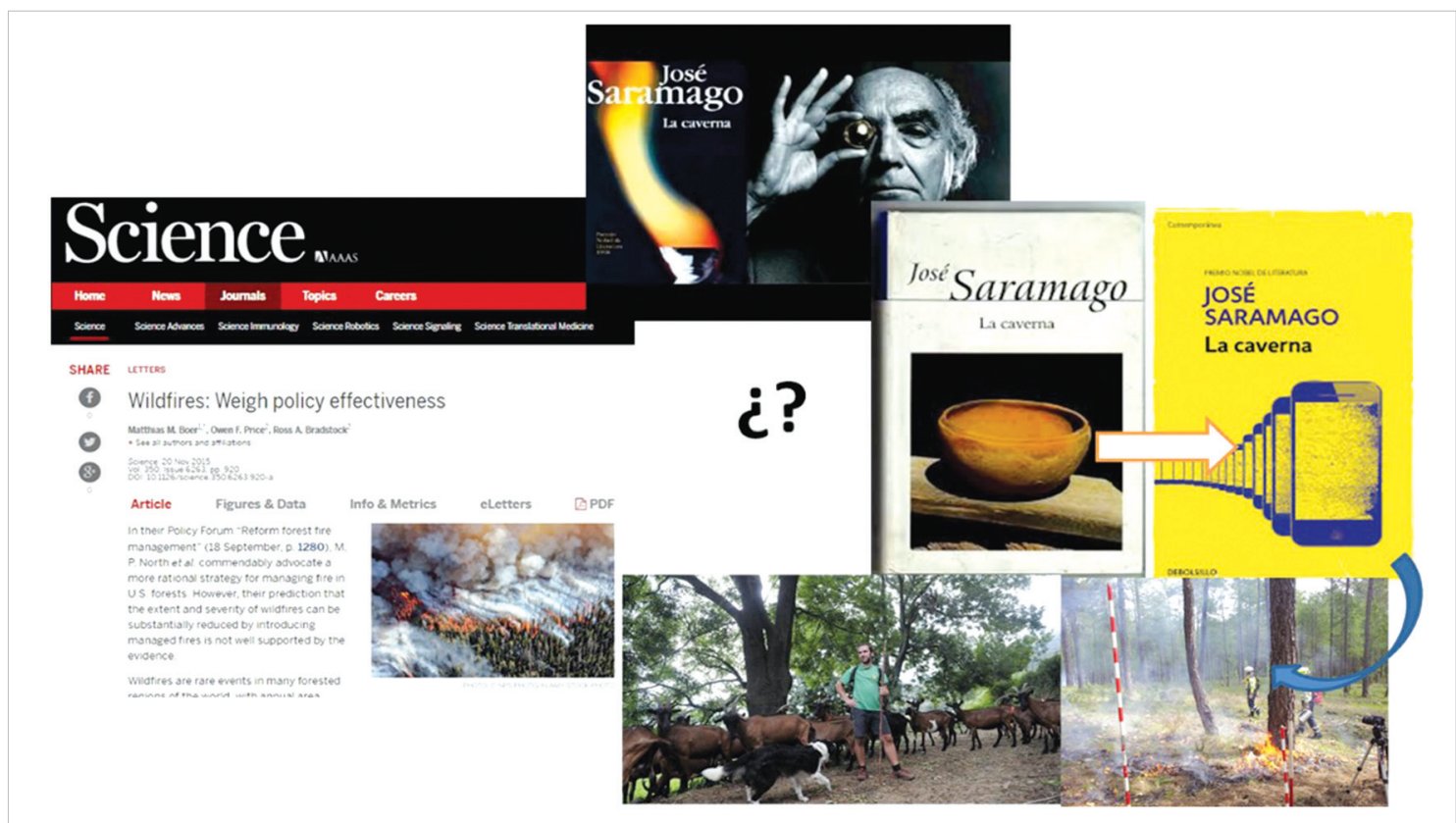

Figura 4. El pesimismo de la comunidad científica ante la eficacia de las medidas preventivas (Boer et al. 2015) parte de que la baja presencia humana en el territorio implica un consumo de combustible a escala paisaje muy por debajo del necesario para limitar los efectos de los grandes incendios en el contexto de Cambio Global. En la Era de la tecnología es necesario "re-inventar" los oficios tradicionales en extensivo para que, al menos, nuestros ecosistemas tengan alguna oportunidad de regenerarse.

\section{La puesta en escena}

Prevenir incendios es una puesta en escena. La música, la danza, el teatro, el cine son artes que nos lo dejan bien claro. Una buena película implica un trabajo de mul- 
titud de personas: guionista, dirección, producción, música, escenografía, vestuario, fotografía, técnicos...y cómo no, los actores y actrices. Esta misma terminología, la de "actores" o "stakeholders", se emplea en los programas de desarrollo rural y efectivamente debe existir una maquinaria bien engrasada para que la eficacia de la prevención sea una realidad. Son legendarias las discusiones entre guionista, director y productor, porque generalmente el que pone el dinero quiere el máximo rédito económico. Si el productor es bueno y le gusta lo que hace, exigirá unos estándares de calidad artística, pero a la vez, querrá que sea una película fácil de visionar por el gran público, vaya al cine y pague su entrada. Moverse entre lo popular y lo artístico nunca ha sido fácil. Nuestros productores son los responsables políticos, los que con su labor se ayudaría a financiar las políticas forestales. Por tanto tenemos buenos guiones, buenos actores, buenos técnicos, pero la puesta en escena nunca llega porque no hay financiación ¿Tiramos la toalla? La mayoría de los proyectos de cine y teatro están agazapados en un cajón hasta que un productor valiente los saca adelante. Puede que en nuestro caso nos cueste encontrar políticos valientes que echen a andar los proyectos de desarrollo rural, que activen de verdad la prevención de incendios a escala de rodal y paisaje, pero el resto de actores seguiremos estando ahí, agazapados, preparados, esperando esa oportunidad y sin parar de llamar a la puerta. A su vez los cambios políticos pueden proceder de demandas sociales, por tanto es necesario que la sociedad entienda las reivindicaciones del sector y que la prevención real implica cambios reales en nuestro mundo rural. En este proceso es necesario destacar a los artesanos de la prevención: agentes forestales, capataces, bomberos forestales y demás profesionales que trabajan todos los días en el monte y que se suelen convertir en artistas en el día a día, en actores secundarios pero no por ello menos importantes, para poner en práctica muchas de las elucubraciones que gestores e investigadores plasmamos en un papel.

La puesta en escena juega un papel muy relevante en la prevención de incendios en la interfaz urbano-forestal (Fig. 5) y urbano-rural, donde nos jugamos vidas humanas y donde frecuentemente fracasan las medidas forestales absorbidas por el suceso de protección civil. Trabajar con rigor y ciencia pero también con sensibilidad artística es fundamental para que la película o la obra de teatro tenga un final feliz.

\section{El arte de comunicar}

El Quijote es nuestro mayor best seller y por algo será. Miguel de Cervantes (1547-1616) supo aunar el arte y el entretenimiento en una novela universal (Cervantes 1605). La coincidencia en el tiempo con la invención de la imprenta facilitó su difusión al gran público y esa fue una gran revolución en las artes, un primer intento por sacar al gran público de la caverna. En la actual Era de la comunicación la revolución es internet y las redes sociales. Como la imprenta, son herramientas, inventos científicos al servicio de la difusión del arte y el conocimiento si se usan adecuadamente. Si en la Era de la comunicación la conexión entre gestores e investigadores sigue siendo ineficaz es porque el problema no está en la herramienta sino en la ac- 


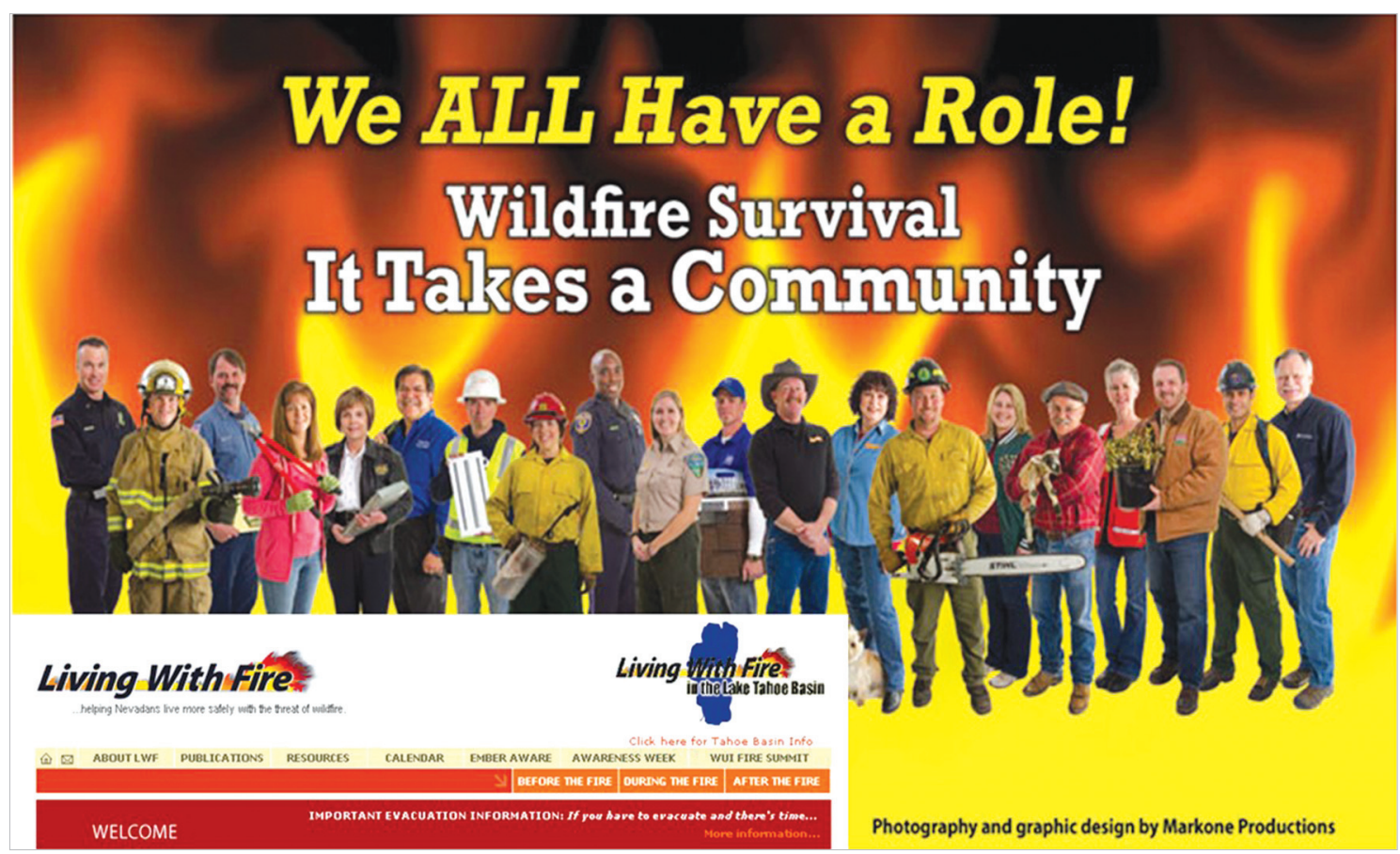

Figura 5. Ejemplo de programa de concienciación en EEUU (http://tahoe.livingwithfire.info/). Implicar a todos los actores en la prevención es una labor fundamental que ya se está poniendo en práctica en Europa en los programas de concienciación y formación en autoprotección en la interfaz urbano-forestal (ver proyecto WUIWATCH https://wuiwatch.org/).

titud. Por otro lado, los científicos forestales, en general hacemos ciencia aplicada, con lo que parece prioritario escuchar a los compañeros que están al pie del cañón para orientar nuestras investigaciones a resolver preocupaciones reales. El papel lo aguanta todo y la creatividad científica tiene sus límites cuando se topa con la realidad de lo cotidiano, por eso es necesario no sólo la comunicación sino la colaboración estrecha entre administraciones de gestión e investigación. Nuestras instituciones no nos lo ponen fácil pero depende de nosotros impulsar estos nexos de unión. Es un ejercicio no remunerado, pero sin duda el más satisfactorio y donde se hace más presente la máxima de Antonio Machado (1875-1939) “Todo necio confunde valor y precio" (Proverbios y Cantares, LXVIII en Machado 2001)

\section{El arte de equivocarse}

Una de las cosas interesantes del arte es que no existe la equivocación, simplemente se puede estar más o menos inspirado en la creación pero no sería correcto (ni justo) decir que una obra artística es "equivocada". En la toma de decisiones en ingeniería del fuego podemos equivocarnos y esa decisión es crítica si implica vidas humanas o desastres ecológicos. Por esas razones la formación, la capacitación, el desarrollo de la carrera profesional, la mejora de protocolos y simulacros, el análisis de 
accidentes y las lecciones aprendidas son herramientas imprescindibles para entender cómo asumir los errores, para aprender a equivocarse.

En definitiva y como dijo el especialista americano en quemas prescritas Kevin Ryan (Ryan 2000), aplicado al empleo del fuego prescrito, pero que se podría extender a cualquier actividad de la prevención de incendios: "La aplicación de quemas prescritas bajo bosques es un arte. Podemos convertirnos en mejores artistas estudiando cuidadosamente el trabajo de grandes artistas y practicando regularmente nuestro arte, pero no seremos nunca artistas perfectos".

\section{Agradecimientos}

Agradezco a la SECF la invitación para presentar esta ponencia en el $7^{\circ}$ Congreso Forestal Español. Mi más sincero agradecimiento a los lectores de Fuegolab que han sido mi apoyo desde 2013 para intentar mejorar en mi labor de divulgación científica y técnica.

\section{Referencias bibliográficas}

Boer, M.M., Price, O.F., Bradstock, R.A., 2015. Wildfires: Weigh policy effectiveness. Sciencemag Letters (Ed. Sills J) Vol 350 issue 6263, 19 November, 2015.

Castillo, M., 2012. Identification and assessment of areas at risk of forest fire using fuzzy methodology. Applied Geography 35 (1-2), 199-207. doi: 10.1016/j.apgeog.2012.07. 001 .

Cervantes, M., 1605. El ingenioso hidalgo Don Qvixote de la Mancha. Disponible on-line http://en.wikipedia.org/wiki/Image:Cervantes_Don_Quixote_1605.gif [Accessed 31/07/ 2017].

Elgammal, A., Saleh, B., 2015. Quantifying Creativity in Art Networks. International Conference on Computational Creativity (ICCC). Disponible online https://arxiv.org/pdf/ 1506.00711.pdf [Accessed 31/07/2017].

Fernandez- Migueláñez, I., Madrigal, J., Garmendia, L., Hernando, C., Guijarro, M., 2014. Aplicación de la lógica fuzzy a la creación áreas cortafuegos compatibles con la extracción de biomasa con fines energéticos. XVII Congreso Español sobre Tecnologías y Lógica Fuzzy STYLF2014. 5-7 febrero 2014. Zaragoza. pp 61-66. Disponible online http://estylf2014.unizar.es/actas.pdf [Accessed 31/07/2017].

Finney, M.A., Cohen, J.D., Forthofer, J.M., McAllister, S.S., Gollner, M.J., Gorham, D.J., Saito, K., Akafuah, N.K., Adam, B.A., English, J.D., 2015. Role of buoyant flame dynamics in wildfire spread. Proceedings of the National Academic of Sciences PNAS, 112 (32): 9833-9838, 1-6. doi: 10.1073/pnas.1504498112

Fisher, P., 2000. Sorites paradox and vague geographies. Fuzzy sets and systems 113, 7-18. doi: 10.1016/S0165-0114(99)00009-3

Gallup, G.G. Jr., 1970. Chimpanzees: self-recognition. Science 167 (3914), 86-87. doi: 10.1126/science.167.3914.86.

Kaplan, J.O., Pfeiffer, M., Kolen, J.C.A., Davis, B.A.S., 2016. Large Scale Anthropogenic Re- 
duction of Forest Cover in Last Glacial Maximum Europe. PLoS ONE 11(11), e0166726. doi: 10.1371/journal.pone.0166726.

Machado, A., 2001. Prosas dispersas (1893-1936). Páginas de Espuma, Madrid (ed. Jordi Doménech). 896 pp. ISBN: 8495642034.

Madrigal. J., Fernández-Migueláñez. I., Hernando. C., Guijarro. M., Vega-Nieva. D., Tolosana. E., 2017. Does forest biomass harvesting for energy reduce fire hazard in the Mediterranean basin? A case study in the Caroig Massif (Eastern Spain). European Journal of Forest Research 136, 13-26. doi: 10.1007/s10342-016-1004-5.

Marino, E., Hernando, C., Planelles, R., Madrigal, J., Guijarro, M., Sebastián, A., 2014. Forest fuel management for wildfire prevention in Spain: a quantitative SWOT analysis. International Journal of Wildland Fire 23, 373-384. doi: 10.1071/WF12203.

Saramago, J., 2000. La caverna. Alfaguara, Madrid, 2000. ISBN 84-204-4228-3.

Platón, 2003. Diálogos. Obra completa en 9 volúmenes. Volumen IV: República. Madrid: Editorial Gredos. ISBN 978-84-249-1027-3.

Regos, A., Aquilué, N., Retana, J., de Cáceres, M., Brotons, L., 2013. Using unplanned fires to help supressing future large fires in Mediterranean forests PLoS ONE 9 (4), e94906. doi: 10.1371/journal.pone.0094906.

Regos, A., Aquilué, N., López, I., Codina, M., Retana, J., Brotons, L., 2016. Synergies between forest biomass extraction for bioenergy and fire suppression in Mediterranean ecosystems: insights from a storyline-and-simulation approach. Ecosystems 19, 1-17. doi: 10.1007/s10021-016-9968-z.

Rothermel, R.C., 1972. A mathematical model for predicting fire spread in wildland fuels. Research paper INT-115, January 1972. Ogden, UT, USDA Forest Service, Intermountain Forest and Range Research Station.

Ryan, K.C., 2000. Técnicas para establecer prescripciones que minimicen o reduzcan la mortalidad del arbolado. Cuadernos de la SECF $\mathrm{n}^{\circ}$ 9, 59-70.

Weir, P., 1989. Deads Poets Society. Touchstone Pictures/Silver Screen Partners IV. Buena Vista Picture Distributions. https://es.wikipedia.org/wiki/Dead_Poets_Society [Accessed 31/07/2017].

Wrangham, R., 2010. Catching Fire: How Cooking Made Us Human. Profile Books LTD. Londres. 312 pp. ISBN: 978-1-84668-286-5. 
